but simultaneously exploits a mathematical loophole to embed the essence of Mottness, the fact that the metal is a doped Mott insulator. The model appears to resolve the conundrum of being a non-Fermi liquid with a Fermi surface in a seemingly unique way. It is very simple: the Pauli-principle counting of the Fermi gas that mandates one electron per spin and site, and the Mottness counting of only one electron per site, can be reconciled by a large contact (Hubbard) interaction that acts only between electrons that have the same momentum. This interaction is microscopically unphysical it is highly non-local in real space - but it does quite well in capturing the qualitative effects of the Mottness, for example in the form of representing upper and lower Hubbard bands in spectral functions $s^{3,9}$.

Phillips and collaborators take this a step further by adding a BCS-style attractive interaction to the model. The result is that it shares qualitative features with BCS $^{3}$ : superconductivity sets in by Cooper instability at a thermal transition that opens a superconducting gap. By construction, the model has a Fermi surface, but this will enclose a different volume in momentum space violating the Luttinger theorem: the Hubbard interaction forces doubly occupied momentum space states into two singly occupied ones in parts of momentum space. Similar logic applies to the Leggett theorem and gives a relation between the normal electron density and superfluid density (shown in Fig. 1b) that is completely different from BCS, showing a very strong suppression of the superfluid density near the density of one electron per site where the Mott insulator appears, reminiscent of the experimental observations.

This construction has at the least the merit of teaching us how to think outside the box of BCS theory, which is much desired given the apparent paradox of the superfluid density $^{6}$. But perhaps we should actually take it more literally. Adiabatic continuity has a remarkable capacity to connect seemingly unrelated entities - for instance, in the ${ }^{3} \mathrm{He}$ liquid the macroscopic free Fermi-gas fixed point is adiabatically continued to the dense van der Waals liquid on the microscopic scale. The barely explored phenomenology of Phillips and collaborators' model may well have more surprises in store ${ }^{3}$ that - by confrontation with reality - may eventually decide if there is a similar continuity between the microscopic Mottness and this non-Fermi liquid with Fermi-surface fixed point.

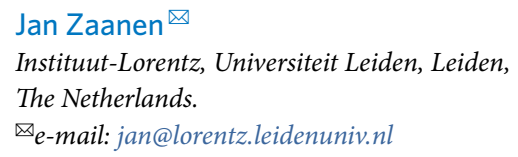

Published online: 27 July 2020

https://doi.org/10.1038/s41567-020-0979-5

References

1. Bardeen, J., Cooper, L. N. \& Schrieffer, J. R. Phys. Rev. 106, 162-164 (1957).

2. Keimer, B., Kivelson, S. A., Norman, M. R., Uchida, S. \& Zaanen, J. Nature 518, 179-186 (2015).

3. Phillips, P. W., Yeo, L. \& Huang, E. W. Nat. Phys. https://doi. org/10.1038/s41567-020-0988-4 (2020).

4. Phillips, P. W. Rev. Mod. Phys. 82, 1719-1742 (2010).

5. Anderson, P. W. The Theory of Superconductivity in the High-T

Cuprate Superconductors (Princeton Univ. Press, 1997).

6. Zaanen, J. Nature 536, 282-283 (2016).

7. Chen, S. D. et al. Science 366, 1099-1102 (2019).

8. Bozovic, I., He, X., Wu, J. \& Bollinger, A. T. Nature 536, 309-311 (2016).

9. Hatsugai, Y. \& Kohmoto, M. J. Phys. Soc. Jpn 61, 2056-2069 (1992).

10. Platé, M. et al. Phys. Rev. Lett. 95, 077001 (2005).

\title{
Facts are relative
}

\author{
The discussion of the quantum mechanical Wigner's friend thought experiment has regained intensity. Recent \\ theoretical results and experimental tests restrict the possibility of maintaining an observer-independent notion of \\ measurement outcomes.
}

\section{Časlav Brukner}

n 1983, Daniel Patrick Moynihan, an American politician, sociologist and diplomat, wrote that "Everyone is entitled to his own opinion, but not his own facts". As much as this may be applied to politics, in quantum mechanics, the objectivity of facts or 'observed events' in the sense that they exist absolutely - and not relative to a particular observer - has recently been challenged in a series of works ${ }^{1-4}$ that build on the Wigner's friend thought experiment ${ }^{5}$. First conceived by physicist Eugene Wigner in 1961, the scenario results in a situation where two observers seem to experience different facts. Writing in Nature Physics, Kok-Wei Bong and co-workers ${ }^{6}$ have now rigorously proved and experimentally demonstrated that a set of plausible assumptions, together with the assumption that an observed event is objective and not relative to anything or anyone, contradicts quantum mechanical predictions and experimental data. The result can be interpreted to imply that in quantum physics, observers are indeed entitled to their own facts.

In the original Wigner's friend thought experiment, an observer in an isolated laboratory - the friend - performs a measurement on a quantum system in an equal superposition of two states. She randomly obtains one of two possible outcomes and updates her system's state to match the observed outcome. The updated state can be verified by repeating the measurement. Meanwhile, a 'superobserver' outside the laboratory - Wigner describes his friend, the laboratory and her system as a large, composite quantum system. He is equipped with instruments of unprecedented precision, which enable him to ascertain the quantum state for the whole laboratory. Most importantly, his measurement that confirms the state does not assign a well-defined value to the outcome of the friend's observation. From Wigner's point of view, his friend exists in a coherent superposition, entangled with the outcome of her measurement. This apparently contradicts the friend's description. Reconciling these two perspectives is at the core of the Wigner's friend paradox. 


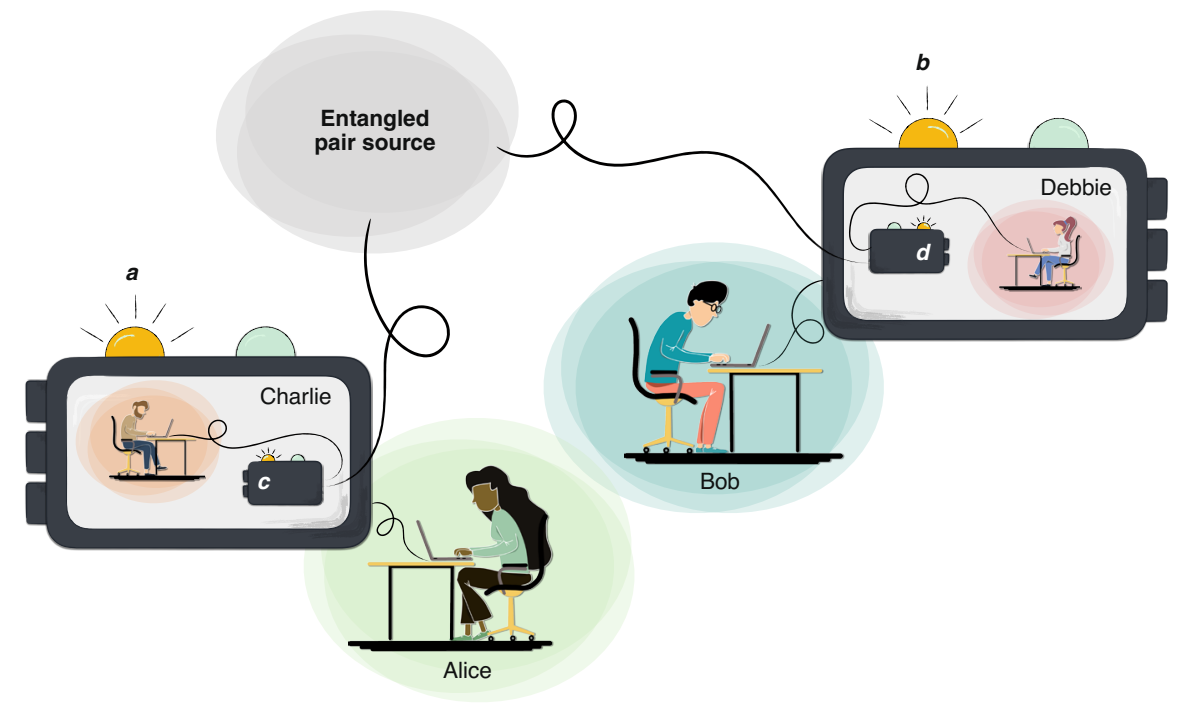

Fig. 1 | The extended Wigner's friend thought experiment. The friends, Charlie and Debbie, are isolated in separate laboratories. They each measure one particle from an entangled pair, obtaining the outcomes $c$ and $d$. Two superobservers, Alice and Bob, are placed outside the laboratories and perform space-like separated measurements with outcomes $a$ and $b$. They choose either to open the laboratories and read out Charlie's and Debbie's outcomes, or to perform large interferometric measurements on the entire laboratories of Charlie and Debbie, respectively. Bong and co-workers ${ }^{5}$ showed that the conjunction of three reasonable assumptions - no-superdeterminism (the choices of measurements are independent of the rest of the experiment), locality (the outcomes are independent of the measurement choice in the distant laboratory) and absoluteness of observed events (an observed event is a real single event, and not relative to anything or anyone) - is in contradiction to quantum theoretical predictions and their proof-of-principle experiment. Consequently, at least one of the assumptions is violated in nature. Credit: G. Rubino.

The observations of Wigner and his friend generate a permanent record in the form of detector clicks or pointer positions. As such, it is tempting to assume they both exist jointly as objective events. Mathematically, this would mean that a joint probability may be assigned to them. It was recently proposed ${ }^{1,2}$ to test this notion by extending the Wigner's friend thought experiment into a scenario to verify the compatibility of quantum mechanics with the idea of local hidden variables ${ }^{7}$ : two separated parties each choose between two or more measurement settings to measure correlations between entangled pairs of particles. Although, in a single run, each party can measure only one observable, with local hidden variables it is possible to assign a joint probability for their full set of potentially measurable observables. If local hidden variables exist, the correlations must be constrained to satisfy the so-called Bell inequalities. Loophole-free experiments have confirmed the violation of the Bell inequalities, invalidating the local hidden variable approach to establishing objective outcomes for all possible measurements ${ }^{8}$.

The extended Wigner's friend thought experiment $^{1,2}$ (Fig. 1) features two spatially separated research laboratories, each with a friend inside (Charlie and Debbie) and a superobserver outside (Alice and Bob, respectively). Charlie and Debbie share an entangled pair of particles, which they measure in their laboratories. Alice and Bob then choose between two measurement settings. They either simply open Charlie's and Debbie's laboratory respectively, asking the friends to report their measurement outcomes, or they follow the original Wigner thought experiment and perform superobserver measurements on the actual laboratories. Importantly, in the second setting all potentially measurable observables are actually measured by four observers. This gives a convincing rationale for the assumption that a joint probability for all four measurements exists. Following the same reasoning as in the local hidden variable approach, the correlations must satisfy the Bell inequalities ${ }^{1,2}$. One of these inequalities, with two measurement settings per party and binary outcomes, was violated in a six-photon experiment ${ }^{4}$, supporting the conclusion that no joint probability exists and the superobservers' and the friends' records are fundamentally incompatible. However, for some measurement settings the argument still assumes that definite outcomes are also assigned to unperformed measurements. This leaves open the possibility of absolute objectivity for actually observed outcomes.

In their new work, Bong and co-workers ${ }^{6}$ started from a set of assumptions that is less restrictive than assuming the existence of local hidden variables (Fig. 1), which they call 'local friendliness', and derived associated inequalities that allow for absolute objectivity without involving outcomes of measurements that were not performed. They proved that the set of local friendliness correlations is a strict superset of the set of local hidden variable correlations, such that it is possible for quantum correlations to violate a Bell inequality while satisfying all the local friendliness inequalities. With only three measurement settings per party and binary outcomes, quantum correlations already violate the local friendliness inequalities. The authors demonstrated the anticipated violation in a proof-of-principle experiment with a pair of photons, where each photon's polarization corresponded to the systems measured by Charlie and Debbie and the photon paths took the roles of Charlie and Debbie themselves.

The argument by Bong and co-workers puts the strongest constraints so far on the possibility that observed facts are absolute, rather than relative to observations or observers. There are interpretations of quantum mechanics that reject the absoluteness of events, as well as those that retain it while violating different assumptions of the argument ${ }^{9}$.

A more conclusive experimental demonstration would require the friend to be a system with the ability to account for a primitive notion of an observer. Unfortunately, this is physically impractical. Extending the experiment from photons to mesoscopic or macroscopic quantum systems would not close the gap unless objectivity of the observed events is restored by a collapse, as postulated by some modifications of quantum mechanics ${ }^{10}$. To develop more convincing tests, the friends might be replaced by systems with increasing levels of complexity, or undergoing thermodynamically irreversible processes, which characterize the redundancy and permanency of physical facts.

That one of our most precise scientific theories might actually be based on subjective facts at its most fundamental level raises deep philosophical questions about the fundamental nature of reality. The hope, however, is that our experiences and decisions in the macroscopic world remain based on objective facts. 


\section{Časlav Brukner, ${ }^{1,2}$ 叫}

${ }^{1}$ Vienna Center for Quantum Science and Technology (VCQ), Faculty of Physics, University of Vienna, Vienna, Austria. ${ }^{2}$ Institute for Quantum Optics and Quantum Information (IQOQI-Vienna), Austrian Academy of Sciences, Vienna, Austria.

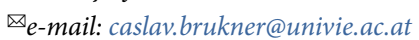

Published online: 17 August 2020

https://doi.org/10.1038/s41567-020-0984-8

References

1. Brukner, C. in Quantum[Un]Speakables II: Half a Century of Bell's Theorem (eds Bertlmann, R. \& Zeilinger, A.) 95-117 (Springer, 2017).

2. Brukner, C. Entropy 20, 350 (2018).

3. Frauchiger, D. \& Renner, R. Nat. Commun. 9, 3711 (2018).
4. Proietti, M. et al. Sci. Adv. 5, eaaw9832 (2019).

5. Wigner, E. P. in The Scientist Speculates (ed. Good, I. J.) 284-302 (Heinemann, 1961).

6. Bong, K.-W. et al. Nat. Phys. https://doi.org/10.1038/s41567-0200990-x (2020).

7. Bell, J. S. Physics 1, 195-200 (1964).

8. Miller, J. L. Phys. Today 69, 14 (2016).

9. Cabello A. in What is Quantum Information? (eds Lombardi, O. et al.) 138-144 (Cambridge Univ. Press, 2017).

10. Bassi, A. \& Ghirardi, G. Phys. Rep 379, 257-426 (2003). 